\title{
A birth population-based survey of preterm morbidity and mortality by gestational age
}

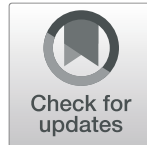

Xiaojing Guo ${ }^{1}$, Xiaoqiong $\mathrm{Li}^{2}$, Tingting $\mathrm{Qi}^{2}$, Zhaojun Pan ${ }^{3}$, Xiaoqin Zhu ${ }^{4}$, Hui Wang ${ }^{4}$, Ying Dong ${ }^{1}$, Hongni Yue ${ }^{3,4^{*}}$ and Bo Sun ${ }^{1 *}$

\begin{abstract}
Background: Despite 15-17 millions of annual births in China, there is a paucity of information on prevalence and outcome of preterm birth. We characterized the outcome of preterm births and hospitalized preterm infants by gestational age (GA) in Huai'an in 2015, an emerging prefectural region of China.

Methods: Of 59,245 regional total births, clinical data on 2651 preterm births and 1941 hospitalized preterm neonates were extracted from Huai'an Women and Children's Hospital (HWCH) and non-HWCH hospitals in 20182020. Preterm prevalence, morbidity and mortality rates were characterized and compared by hospital categories and GA spectra. Death risks of preterm births and hospitalized preterm infants in the whole region were analyzed with multivariable Poisson regression.

Results: The prevalence of extreme, very, moderate, late and total preterm of the regional total births were 0.14 , $0.53,0.72,3.08$ and 4.47\%, with GA-specific neonatal mortality rates being 44.4, 15.8, 3.7, 1.5 and 4.3\%, respectively. There were 1025 (52.8\% of whole region) preterm admissions in HWCH, with significantly lower in-hospital death rate of inborn (33 of 802, 4.1\%) than out-born (23 of 223, 10.3\%) infants. Compared to non-HWCH, three-fold more neonates in HWCH were under critical care with higher death rate, including most extremely preterm infants. Significantly all-death risks were found for the total preterm births in birth weight $<1000 \mathrm{~g}$, GA $<32$ weeks, amniotic fluid contamination, Apgar-5 min $\leq 7$, and birth defects. For the hospitalized preterm infants, significantly in-hospital death risks were found in out-born of HWCH, GA $<32$ weeks, birth weight $<1000 \mathrm{~g}$, Apgar-5 min $\leq 7$, birth defects, respiratory distress syndrome, necrotizing enterocolitis and ventilation, whereas born in HWCH, antenatal glucocorticoids, cesarean delivery and surfactant use decreased the death risks.
\end{abstract}

Conclusions: The integrated data revealed the prevalence, GA-specific morbidity and mortality rate of total preterm births and their hospitalization, demonstrating the efficiency of leading referral center and whole regional perinatalneonatal network in China. The concept and protocol should be validated in further studies for prevention of preterm birth.

Keywords: Birth population, Prevalence, Preterm, Morbidity, Mortality, Gestation, Regional perinatal-neonatal network

\footnotetext{
* Correspondence: yuehongni@hotmail.com; bsun@shmu.edu.cn;

bsun9898@163.com

${ }^{3}$ Department of Neonatology and Unit of Population Health Information,

Huai'an Women and Children's Hospital, 104 South Ren Min Road, Huai'an

223002, Jiangsu, China

${ }^{1}$ The NCH Key Laboratory of Neonatal Diseases, National Children's Medical

Center, Children's Hospital of Fudan University, Shanghai 201102, China

Full list of author information is available at the end of the article
}

\section{$\triangle B M C$}

(c) The Author(s). 2021, corrected publication 2021. Open Access This article is licensed under a Creative Commons Attribution 4.0 International License, which permits use, sharing, adaptation, distribution and reproduction in any medium or format, as long as you give appropriate credit to the original author(s) and the source, provide a link to the Creative Commons licence, and indicate if changes were made. The images or other third party material in this article are included in the article's Creative Commons licence, unless indicated otherwise in a credit line to the material. If material is not included in the article's Creative Commons licence and your intended use is not permitted by statutory regulation or exceeds the permitted use, you will need to obtain permission directly from the copyright holder. To view a copy of this licence, visit http://creativecommons.org/ licenses/by/4.0/. The Creative Commons Public Domain Dedication waiver (http://creativecommons.org/publicdomain/zero/1. 0/) applies to the data made available in this article, unless otherwise stated in a credit line to the data. 


\section{Background}

Perinatal and neonatal morbidity and mortality of preterm births represent the focus of quality improvement with perinatal healthcare strategies [1-5]. Despite the majority of neonatal deaths attributed to preterm birth complication, and a large amount of hospital admissionbased data by gestational age (GA), available in China, the whole picture of perinatal-neonatal care remains unclear due to a lack of birth population-based clinical surveys from obstetric and neonatal perspective [6-9]. Although the number of Chinese annual birth population has reached 15-17 millions in the past decade, with an established universal health insurance covering maternal-infant healthcare [10-13], there is a paucity of data on vital statistics of preterm births and assessment of combined effects of antenatal, peripartum and postnatal interventions on perinatal and neonatal outcome in the preterm birth and hospitalized population.

Many institute-based surveys and controlled studies may provide prevalence, morbidity and mortality as outcome of hospitalized preterm population or samples, however, reliable information in birth population-based outcome of hospitalized neonatal infants remains lacking from China. For prevention of preterm birth, perinatal and neonatal death, many efforts have been exerted in the past decade with the universal maternal-infant health insurance coverage as well as facility-based robust technologies, including antenatal care with fetal ultrasound monitoring, glucocorticosteroids, tocolysis, delivery resuscitation and neonatal intensive and critical care $[1-5$, $7,8,14-16]$. However, efficacy of these strategies may vary taking geographic and socioeconomic status of China into account. In this regard, further in-depth investigations are required to unravel the efficiency of perinatal-neonatal service as an integrated antenatal, peripartum and postnatal care system under particular clinical settings and infrastructure, in order to optimize facility-based practice in emerging regions in world-wide perspective.

The national surveillance system reporting on perinatal information in China has limitations as it only retrieved data of preterm births $\geq 28$ weeks of GA [6, 1719]. Huai'an is an emerging prefectural region in east China with 5.6-million population and 50\% rural residents, the regional gross domestic production per capita approaching the national average level. According to our previous surveys of complete birth registries in Huai'an in 2015 , a survival rate up to 44 weeks of adjusted postmenstrual age (aPMA) of more than 50\% was observed in preterm infants with 27-28 gestational weeks and beyond [11]. By comparison, the $50 \%$ survival rate was reported in 23-25 weeks of gestation from the nation-wide registry or perinatal network data in developed countries [1-5, 7-9]. Our previous data demonstrated substantial and persistent improvement in Huai'an perinatalneonatal care between 2010 and 2015, probably due to the establishment of centralized prenatal care and deliveries at level II and III hospitals [10, 11, 20]. This enabled us to take facility-specific conditions and regional infrastructure into consideration, when investigating the impact of integrated antenatal, peripartum and postnatal interventions on preterm especially extreme preterm survival in this region.

The current study aimed to delineate the prevalence, morbidity and mortality of whole regional preterm births and hospitalized infants stratified by GA, to investigate the role of Huai'an Women and Children's Hospital $(\mathrm{HWCH})$ as the main transferal center of whole region, and to verify the efficacy of perinatal interventions and the underlying neonatal morbidities of deaths. As birth population-based studies on perinatal and neonatal healthcare are still at preliminary stage in China $[6,12$, 13], our concept and methodology of using regional birth registry data to assess death risks in hospitalized preterm population, and the quality of regional perinatal-neonatal healthcare system, may be helpful to re-evaluate and optimize the standard of care and health insurance policy in the emerging region in transition of China $[6,13]$.

\section{Methods}

Study population, protocol and ethical approval

The concept and protocol of investigation, definition of diagnosis of preterm morbidity and mortality as well as specific disease severity and level of care were adopted from the references cited (see below), during the data collection, analysis and preparation of manuscript in 2018-2020. This current cross-sectional study followed the whole regional birth population survey in 2015, in which a complete birth data was prospectively collected from totally 107 level I-III hospitals providing obstetric care (6 municipal, 16 county and 85 township), and 8 level II and 4 level III hospitals equipped with neonatal wards and/or intensive care units (NICU) [11, 20]. Nonmedical abortions (especially unplanned pregnancy) were excluded. The above birth data was integrated with the data on all hospitalized preterm infants derived from the complete birth population in whole region, retrospectively retrieved from regional perinatal information database (Fig. 1). The study protocol was approved by the ethic committee of Children's Hospital of Fudan University, and accepted by $\mathrm{HWCH}$ as well as all participating hospitals in Huai'an $[11,20]$. As no specific intervention was applied, informed consent from parents/ guardians was waived. Preterm birth was defined as delivery at $25^{+0}-36^{+6}$ weeks of GA, divided into extreme (EPT, $25^{+0}-27^{+6}$ weeks), very (VPT, $28^{+0}-31^{+6}$ weeks), moderate (MPT, $32^{+0}-33^{+6}$ weeks) and late (LPT, 
$34^{+0}-36^{+6}$ weeks) preterm groups [21, 22]. Those of EPT below 25 weeks of GA were not included due to very few numbers and parental decision not to provide resuscitation at delivery. Incidences of EPT, VPT, MPT, LPT and total preterm births were presented as prevalence in percent of the number of total births (including term and post-term births) of whole region.

\section{Definitions of preterm morbidity and mortality}

The diagnostic criteria of pregnancy, perinatal and neonatal co-morbidities and complications are presented in additional file 1 [23-34]. Definitions regarding vital statistics are based on the original survey [11, 20], and the 10th revision of the international classification of diseases [35]. Briefly, GA was mainly determined by the date of last menstrual period and/or fetal sonography in early pregnancy, or postnatal assessment by new Ballard score when prenatal records were missing or incomplete [36]. Birth weight (BW) was measured at birth. Small for GA (SGA) was defined as a $\mathrm{BW}<10$ th percentile for gender and $\mathrm{GA}$ [37]. Birth defects (BD) were identified prenatally or within the first 7 postnatal days (PND) [11, 20]. Severity of neonatal underlying diseases were characterized as requiring intensive or critical care based on the treatment strength during hospitalization, such as degree of the disease severity, in need of non-invasive / mechanical ventilation (NIV/MV), surfactant, vasopressor, or surgery, etc. (also see additional file 1) [7, $28,38]$. Fetal death was deemed to be synonymous with stillbirth. Deaths at delivery room (DR) referred to those born alive but died during resuscitation,

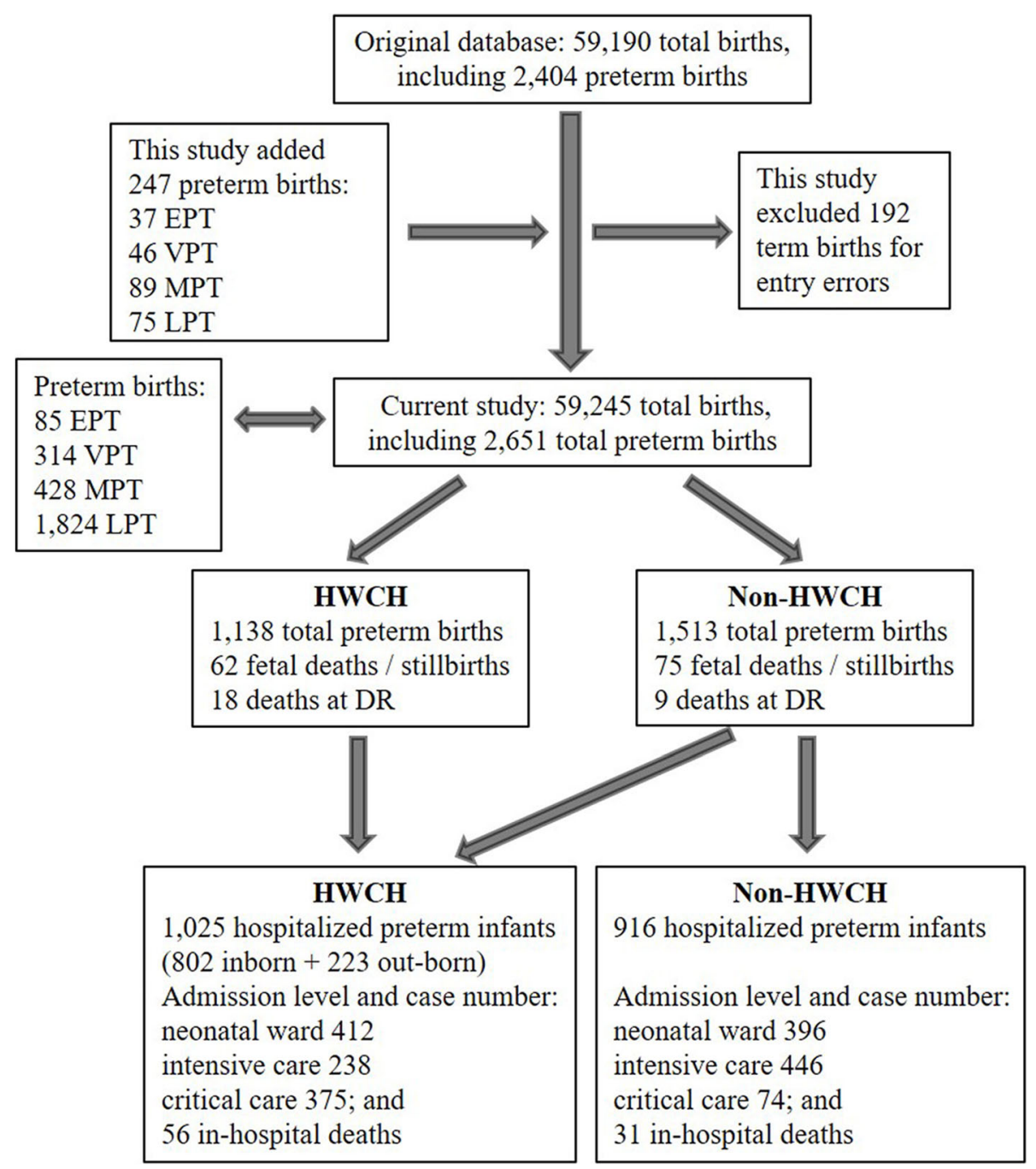

Fig. 1 The flowchart of database and enrolled preterm population by gestational age strata and hospital categories. Abbreviations: EPT, extremely preterm; VPT, very preterm; MPT, moderate preterm; LPT, late preterm; ET, early term. For definitions of deaths and other abbreviations see Table 1 legends 
usually after parents' requests for withdrawal or withholding [39]. Perinatal mortality included stillbirths and neonatal deaths within first 7 PND. Neonatal mortality was defined as deaths of livebirths within 28 PND, including deaths at DR. All-death was defined by including stillbirths, neonatal deaths or deaths within 44 weeks of aPMA for EPT and VPT. Inhospital death was defined as deaths during hospitalization within 28 PND, or within 44 weeks of aPMA for EPT and VPT. For those hospitalized in NICU but had early withdrawal of treatment at parental request, the outcome was estimated by attending physicians based on discharge or follow-up record.

In birth population, prevalence of perinatal and neonatal morbidities was presented as percentage (\%) with the numbers of preterm total births and livebirths excluding deaths at DR as denominator (unless otherwise specified). In the hospitalized population, prevalence of postnatal morbidities was presented as percentage (\%) with the number of total preterm admissions as the denominator. Rates (\%) of perinatal mortality, stillbirths, deaths at DR and all-death were divided by the number of total preterm births. Neonatal mortality rate (\%) was divided by the number of preterm livebirths (including deaths at DR) [20]. In-hospital death rates (\%) were divided by the number of preterm livebirths excluding deaths at DR in birth population, or the number of total preterm admissions in hospitalized population, respectively. The GA-specific mortality rates (\%, where numerators and denominators were both limited within specific GA stratum) [7, 40] were adopted and corrected by the number of total births or livebirths of whole region [41], and presented as per 1000 (\%, for calculation see Table 2).

\section{Representativeness of $\mathrm{HWCH}$ and risk factors of deaths}

To estimate the representativeness of $\mathrm{HWCH}$ of whole region, whole regional preterm births and hospitalized preterm infants were divided into $\mathrm{HWCH}$ and non$\mathrm{HWCH}$ groups, respectively. Furthermore, the $\mathrm{HWCH}$ admissions were divided into inborn and out-born to compare the efficacy of perinatal-neonatal care by hospital categories (Fig. 1). Risks of all-death for preterm births, and in-hospital death for preterm admissions, were analyzed to determine the role of $\mathrm{HWCH}$ in the whole region, taking the effects of perinatal interventions and the underlying neonatal morbidities into consideration.

\section{Statistical analysis}

EPIDATA database was used for datasheet recordings and subjected to SPSS software (V. 16.0, SPSS Inc. Chicago, IL) for statistical analysis. Continuous variables were presented as mean and standard deviation (SD) or median [interquartile ranges]. One-way analysis of variance or non-parametric Mann-Whitney test was used for comparison among groups. Categorical variables were presented as number and rate, using a two-tailed Pearson Chi-squared or Fisher's exact test where appropriate. $P<0.05$ was considered statistically significant. Death risks of preterm births and hospitalizations were analyzed by uni- and multi-variable Poisson regressions by including major risk factors in terms of admission hospital categories, pregnancy complications, perinatal conditions and preterm co-morbidities as covariates. Values were given as relative risks (RR) with 95\% confidence intervals $(\mathrm{CI})$, or as crude and adjusted odds ratio (OR) with 95\% CI for GA strata with Mantel-Haenszel Chi-square test, where appropriate.

\section{Results}

\section{Preterm morbidity and mortality of whole region}

Of the 59,245 regional total births (and 59,056 livebirths), 2651 (4.5\%) were preterm in which 2527 women had fetal sonography early in pregnancy, with preterm perinatal and neonatal mortality rates being 8.4 and $4.3 \%$, respectively (Table 1 ). The prevalence of EPT, VPT, MPT, LPT and total preterm of the total births of whole region were $0.14,0.53,0.72,3.08$ and $4.47 \%$, with corresponding all-death rate 1.0, 1.6, 0.6, 1.1 and 4.3\%o, respectively (Table 2 ), and GA-specific neonatal mortality rate $44.4,15.8,3.7,1.5$ and $4.3 \%$, respectively. The GA-specific all-death rates of 25, 26, 27 and 28 weeks were $100,78.1,56.4$ and $52.1 \%$, and in-hospital death rates $100,70.0,48.3$ and $30.4 \%$, with a $50 \%$ survival rate achieved by 28 and 27 week of GA, respectively (Fig. 2).

Of 2487 preterm livebirths excluding deaths at DR, the hospitalization and in-hospital death rate was 78.0 and $3.5 \%$, respectively (Table 1 ). In 1941 hospitalized preterm infants (68 had GA determined by new Ballard score), 35.2 and $23.1 \%$ received intensive and critical care (Table 3), with in-hospital death rates being $0.6 \%$ $(4 / 684)$ and $18.5 \%$ (83/449), respectively. The top five morbidities in hospitalized preterm infants were hyperbilirubinemia (59.0\%), pneumonia/sepsis (40.0\%), respiratory distress syndrome (RDS) (14.1\%), intraventricular hemorrhage $(11.0 \%)$ and hypoglycemia (10.8\%), respectively. Surfactant and NIV/MV were applied in 11.2 and $39.9 \%$ of the hospitalized preterm infants, respectively (Table 3).

\section{Representativeness of $\mathrm{HWCH}$}

In the 59,245 total births of whole region, 9405 (15.9\%) were born in $\mathrm{HWCH}$, with significantly higher rates of major pregnancy complications and preterm birth (> threefold) than those of non-HWCH hospitals (Table 1). Of the whole region, proportions of births and all-deaths for EPT in $\mathrm{HWCH}$ were 71.8 and $78.7 \%$, respectively. A total of 
Table 1 Perinatal demographic status, prevalences and mortality rates of preterm births in Huai'an in 2015

\begin{tabular}{|c|c|c|c|}
\hline & $\mathrm{HWCH}$ & Non-HWCH & Whole region \\
\hline Total births & 9405 & 49,840 & 59,245 \\
\hline Total live births & 9328 & 49,728 & 59,056 \\
\hline Preterm births $s^{1 * * *}$ & $1138(12.1)$ & $1513(3.0)$ & $2651(4.5)$ \\
\hline Preterm livebirths & $1076(94.6)$ & 1438 (95.0) & $2514(94.8)$ \\
\hline $\mathrm{HDP} * * *$ & $253(22.2)$ & $182(12.0)$ & $435(16.4)$ \\
\hline $\mathrm{GDM}^{* * *}$ & $102(9.0)$ & $21(1.4)$ & $123(4.6)$ \\
\hline Anemia*** & $287(25.2)$ & $88(5.8)$ & $375(14.1)$ \\
\hline PROM & $377(33.1)$ & $446(29.5)$ & $823(31.0)$ \\
\hline $\mathrm{ANG}^{* *}$ & $513(45.1)$ & $596(39.4)$ & $1109(41.8)$ \\
\hline Cesarean delivery* & $685(60.2)$ & $851(56.2)$ & $1536(57.9)$ \\
\hline Male & $649(57.0)$ & $865(57.2)$ & $1514(57.1)$ \\
\hline GA, weeks** & $33.8 \pm 2.8$ & $34.1 \pm 2.3$ & $34.0 \pm 2.5$ \\
\hline $25-27^{* * *}$ & $61(5.4)$ & $24(1.6)$ & $85(3.2)$ \\
\hline $28-31$ & $132(11.6)$ & $182(12.0)$ & $314(11.8)$ \\
\hline $32-33$ & $173(15.2)$ & $255(16.9)$ & $428(16.1)$ \\
\hline $34-36$ & $772(67.8)$ & 1052 (69.5) & $1824(68.8)$ \\
\hline BW, g & $2369 \pm 656$ & $2420 \pm 657$ & $2398 \pm 657$ \\
\hline$<1000^{* * *}$ & $57(5.0)$ & $12(0.8)$ & $69(2.6)$ \\
\hline $1000-1499$ & $85(7.5)$ & $118(7.8)$ & $203(7.7)$ \\
\hline $1500-2499$ & $467(41.0)$ & $616(40.7)$ & $1083(40.9)$ \\
\hline$\geq 2500^{*}$ & $529(46.5)$ & $767(50.7)$ & $1296(48.9)$ \\
\hline Multiple births*** & $311(27.3)$ & $294(19.4)$ & $601(22.8)$ \\
\hline SGA & $47(4.1)$ & $70(4.6)$ & $117(4.4)$ \\
\hline AF contamination* & $72(6.3)$ & $128(8.5)$ & $200(7.5)$ \\
\hline Grade II & $8(0.7)$ & $27(1.8)$ & $35(1.3)$ \\
\hline Grade III & $48(4.2)$ & $50(3.3)$ & $98(3.7)$ \\
\hline Apgar $1-\min \leq 7^{* * *}$ & $472(41.5)$ & $278(18.4)$ & $750(28.3)$ \\
\hline Apgar $5-\min \leq 7^{* * *}$ & $175(15.4)$ & $156(10.3)$ & $331(12.5)$ \\
\hline Birth defects & $36(3.2)$ & $45(3.0)$ & $81(3.1)$ \\
\hline Hospitalization ${ }^{2 *}$ & $802(75.8)$ & $1139(79.7)$ & $1941(78.0)$ \\
\hline Perinatal mortality ${ }^{3}$ & $101(8.9)$ & $123(8.1)$ & $224(8.4)$ \\
\hline Fetal deaths/stillbirths & $62(5.4)$ & $75(5.0)$ & $137(5.2)$ \\
\hline Deaths at DR* & $18(1.6)$ & $9(0.7)$ & $27(1.0)$ \\
\hline Neonatal mortality ${ }^{4}$ & $49(4.6)$ & $58(4.0)$ & $107(4.3)$ \\
\hline In-hospital deaths ${ }^{5}$ & $33(3.1)$ & $54(3.8)$ & $87(3.5)$ \\
\hline All-death ${ }^{6}$ & $114(10.0)$ & $138(9.1)$ & $252(9.5)$ \\
\hline
\end{tabular}

Abbreviations: HWCH, Huai'an Women and Children's Hospital; non-HWCH, hospitals excluding HWCH; HDP, hypertensive disorder of pregnancy; GDM, gestational diabetes mellitus; PROM, prelabor rupture of membrane; ANG, antenatal glucocorticoids; GA, gestational age; BW, birth weight; SGA, small for gestational age; $\mathrm{AF}$, amniotic fluid; $\mathrm{DR}$, delivery room

Values are given in $n, n(\%)$ or means + SD. Percentage (\%) refers to number of preterm births in respective columns unless otherwise stated. ${ }^{*} P<0.05$, ** $P<0.01$ and ${ }^{* * *} P<0.001$ for HWCH vs. non-HWCH

1. Percentage (\%) refers to the number of total births (including term and post term births) in respective columns

2. Percentage (\%) refers to number of preterm livebirths excluding deaths at DR in respective columns

3. Perinatal mortality includes preterm fetal deaths / stillbirths from 25 complete gestational week till deaths within the first 7 postnatal days (including deaths at DR)

4. Neonatal mortality includes preterm deaths at DR and of livebirths within 28 postnatal days. Percentage (\%) refers to number of preterm livebirths in respective columns

5. In-hospital deaths include preterm deaths of hospitalized infants until 44 week of adjusted post menstrual age (excluding deaths at DR). Percentage (\%) refers to above note 2 for definition

6. All-death includes preterm fetal deaths / stillbirths and postnatal deaths from 25 complete gestational week till 44 week of adjusted post menstrual age 
Table 2 Constitution of GA-specific mortality rates or corrected by the total births of whole region

\begin{tabular}{|c|c|c|c|c|c|}
\hline GA, weeks & $25-27$ & $28-31$ & $32-33$ & $34-36$ & $25-36$ \\
\hline Preterm births, $\mathrm{n}$ & 85 & 314 & 428 & 1824 & 2651 \\
\hline Prevalence, $\%{ }^{1}$ & 1.4 & 5.3 & 7.2 & 30.8 & 44.7 \\
\hline Perinatal mortality, $\mathrm{n}$ & 49 & 83 & 32 & 60 & 224 \\
\hline $\mathrm{R} 1, \%$ & 57.6 & 26.4 & 7.5 & 3.3 & 8.4 \\
\hline$R 2, \%$ & 0.8 & 1.4 & 0.5 & 1.0 & 3.8 \\
\hline Fetal deaths / stillbirths, n & 31 & 49 & 20 & 37 & 137 \\
\hline $\mathrm{R} 1, \%$ & 36.5 & 15.6 & 4.7 & 2.0 & 5.2 \\
\hline $\mathrm{R} 2, \%$ & 0.5 & 0.8 & 0.3 & 0.6 & 2.3 \\
\hline Deaths at $D R, n$ & 12 & 11 & 2 & 2 & 27 \\
\hline $\mathrm{R} 1, \%$ & 14.1 & 3.5 & 0.5 & 0.1 & 1.0 \\
\hline$R 2, \%$ & 0.2 & 0.2 & 0.0 & 0.0 & 0.5 \\
\hline Neonatal mortality, $\mathrm{n}$ & 24 & 42 & 15 & 26 & 107 \\
\hline $\mathrm{R} 1, \%^{2}$ & 44.4 & 15.8 & 3.7 & 1.5 & 4.3 \\
\hline$R 2, \% 0^{3}$ & 0.4 & 0.7 & 0.3 & 0.4 & 1.8 \\
\hline All deaths, $n$ & 61 & 93 & 35 & 63 & 252 \\
\hline $\mathrm{R} 1, \%$ & 71.8 & 29.6 & 8.2 & 3.5 & 9.5 \\
\hline $\mathrm{R} 2, \% \mathrm{o}$ & 1.0 & 1.6 & 0.6 & 1.1 & 4.3 \\
\hline
\end{tabular}

Abbreviations: GA, gestational age; DR, delivery room. Values are number (n) or rate. GA-specific mortality rate $(\mathrm{R} 1, \%)=($ number of deaths of each GA stratum / number of births of the same GA stratum) $\times 100$; GA-specific mortality rate corrected by the number of total births (including term and post term births) of whole region $(R 2, \%)=G A$-specific mortality rate $\times$ prevalence of each GA in the total births = (number of deaths of each GA stratum / the number of total births $[59,245]) \times 1000$. For definition of all-death see Table 1 legends, note 6

1. Prevalences of each GA in the total births $(\% 0)=$ (number of births of each GA stratum / the number of total births) $\times 1000$

2. R1, calculated by number of neonatal deaths of each GA stratum divided by number of livebirths in the same GA stratum

3. R2, calculated by number of neonatal deaths of each GA stratum divided by the number of livebirths (including term and post term births) of whole region $(59,056)$
1025 (52.8\% of whole region) preterm infants were hospitalized in HWCH (802 inborn and 223 out-born). The inhospital death rate was significantly higher in the out-born than in the inborn infants, especially within 7 PND, and in VPT and LPT (Table 3). Compared to non-HWCH, the rate of hospitalized preterm infants received critical care was four times as high as in $\mathrm{HWCH}$, with significantly longer length of stay, higher costs, and higher rates of inhospital deaths and major morbidities (Table 3).

\section{Uni- and multi-variable Poisson regression analysis}

In general, for the variables with moderate to high relative risks of deaths by univariable regression analysis, it tended to become mild or no risk by multivariable regression model. The effect of mitigation of risks was also seen in stratified analysis by GA (Table S1). Significantly independent all-death risks were found for the total preterm births in $\mathrm{GA}<32$ weeks, $\mathrm{BW}<1000 \mathrm{~g}$, amniotic fluid contamination, Apgar-5 min $\leq 7$, and BD (Table 4a). For the hospitalized preterm infants, significantly inhospital death risks were found in the out-born of HWCH, GA < 32 weeks, BW < 1000 g, Apgar-5 min $\leq 7$, $\mathrm{BD}$, RDS, necrotizing enterocolitis and NIV/MV (Table 4b), whereas born in $\mathrm{HWCH}$, antenatal glucocorticoids, cesarean delivery and surfactant use reduced death risks (Table $4 \mathrm{a}$ and $\mathrm{b}$ ).

\section{Discussions}

Our study demonstrated the prevalences, perinatal and neonatal morbidity and mortality by GA strata of total preterm births or hospitalized preterm infants, from a complete birth population in an emerging region of China. These findings should be complementary to the findings from reports of national surveys by sampling, or institution-based studies on perinatal and neonatal outcome in China, which lacked comprehensive perinatal data from the regional birth-population perspective [42-
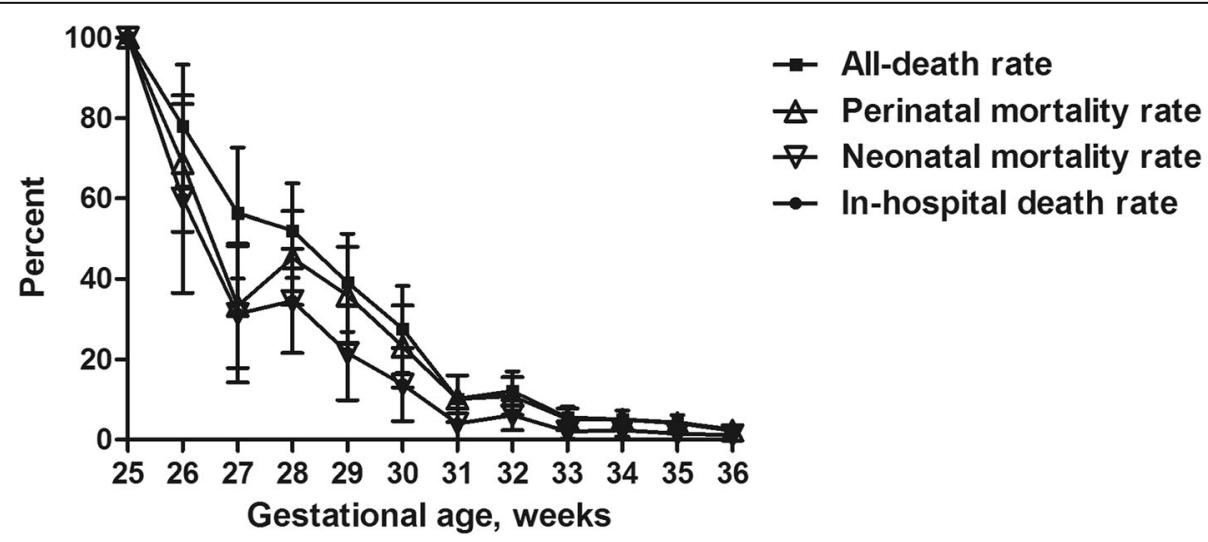

Fig. 2 The all-death rate, perinatal mortality rate, neonatal mortality rate and in-hospith
each gestational age. Legends: For definitions of death rates, see Table 1 footnotes 
Table 3 Perinatal status, morbidity and mortality rates of all hospitalized preterm infants in Huai'an

\begin{tabular}{|c|c|c|c|c|c|}
\hline & $\mathrm{HWCH}$ & & & Non-HWCH & Whole \\
\hline & Inborn & Out-born & Total & hospitals & region \\
\hline Hospitalization & 802 & 223 & 1025 & 916 & 1941 \\
\hline Intensive care & $184(22.9)$ & $54(24.2)$ & $238(23.2)$ & 446 (48.7)\#\#\# & $684(35.2)$ \\
\hline Critical care $^{1}$ & $283(35.3)$ & $92(41.3)$ & $375(36.6)$ & 74 (8.1)\#\#\# & $449(23.1)$ \\
\hline Length of stay, days & $14[10,19]$ & $14[9,21]$ & $14[10,20]$ & $8[5,13] \# \# \#$ & $11[7,17]$ \\
\hline Costs, $\times 1000$ CNY & $13[9,20]$ & $13[9,23]$ & $13[9,20]$ & $7[4,12] \# \# \#$ & $10[6,17]$ \\
\hline Cesarean delivery & $509(63.5)$ & $104(46.6)^{* * *}$ & $613(59.8)$ & $556(60.7)$ & $1169(60.2)$ \\
\hline Male & $472(58.9)$ & $127(57.0)$ & $599(58.4)$ & $496(54.1)$ & $1095(56.4)$ \\
\hline GA, weeks & $33.7 \pm 2.4$ & $33.6 \pm 2.5$ & $33.7 \pm 2.4$ & $34.1 \pm 2.1 \# \# \#$ & $33.9 \pm 2.3$ \\
\hline $25-27$ & $21(2.6)$ & $7(3.1)$ & $28(2.7)$ & 12 (1.3)\# & $40(2.1)$ \\
\hline $28-31$ & 109 (13.6) & $34(15.2)$ & $143(14.0)$ & $98(10.7)$ & $241(12.4)$ \\
\hline $32-33$ & $160(20.0)$ & $44(19.7)$ & $204(19.9)$ & $155(16.9)$ & $359(18.5)$ \\
\hline $34-36$ & $512(63.8)$ & $138(61.9)$ & $650(63.4)$ & 651 (71.1)\#\# & $1301(67.0)$ \\
\hline BW, g & $2280 \pm 610$ & $2270 \pm 625$ & $2278 \pm 614$ & $2376 \pm 636 \# \#$ & $2324 \pm 626$ \\
\hline$<1000$ & $12(1.5)$ & $1(0.4)$ & $13(1.3)$ & $7(0.8)$ & $20(1.0)$ \\
\hline 1000-1499 & $72(9.0)$ & $27(12.1)$ & $99(9.7)$ & $73(8.0)$ & $172(8.9)$ \\
\hline $1500-2499$ & $403(50.2)$ & $110(49.3)$ & $513(50.0)$ & $422(46.1)$ & $935(48.2)$ \\
\hline$\geq 2500$ & 315 (39.3) & $85(38.1)$ & $400(39.0)$ & 414 (45.2)\#\# & $814(41.9)$ \\
\hline Multiple births & $214(26.7)$ & $35(15.7)^{* *}$ & $249(24.3)$ & 140 (15.0)\#\#\# & $389(20.0)$ \\
\hline SGA & $35(4.4)$ & $12(5.4)$ & $47(4.6)$ & $50(5.5)$ & $97(5.0)$ \\
\hline AF contamination & $39(4.9)$ & $15(6.7)$ & $54(5.3)$ & $66(7.2)$ & $120(6.2)$ \\
\hline Grade II & $5(0.6)$ & $4(1.8)$ & $9(0.9)$ & $17(1.9)$ & $26(1.3)$ \\
\hline Grade III & $24(3.0)$ & $9(4.0)$ & $33(3.2)$ & $26(2.8)$ & $59(3.0)$ \\
\hline Apgar $1-\min \leq 7$ & $375(46.8)$ & $42(18.8)^{* * *}$ & $417(40.7)$ & 156 (17.0)\#\#\# & $573(29.5)$ \\
\hline Apgar 5 -min $\leq 7$ & $91(11.3)$ & $24(10.8)$ & $115(11.2)$ & 52 (5.7)\#\#\# & $167(8.6)$ \\
\hline Birth defects & $26(3.1)$ & $20(9.0)^{* * *}$ & $45(4.4)$ & 109 (11.9)\#\#\# & $154(7.9)$ \\
\hline Admitted within $24 \mathrm{~h}$ & $711(88.7)$ & $145(65.0)^{* * *}$ & $856(83.5)$ & 712 (77.7)\#\# & $1568(80.8)$ \\
\hline $\mathrm{IVH}$ & $99(12.3)$ & $24(10.8)$ & $123(12.0)$ & $90(9.8)$ & $213(11.0)$ \\
\hline RDS & $119(14.8)$ & $38(17.0)$ & $157(15.3)$ & $116(12.7)$ & $273(14.1)$ \\
\hline Pneumonia / sepsis & $367(45.8)$ & $116(52.0)$ & $483(47.1)$ & 294 (32.1)\#\#\# & $777(40.0)$ \\
\hline Hypoglycemia & $148(18.5)$ & $31(13.9)$ & 179 (17.5) & 30 (3.3)\#\#\# & 209 (10.8) \\
\hline PDA & $11(1.4)$ & $4(1.8)$ & $15(1.5)$ & $4(0.4) \#$ & $19(1.0)$ \\
\hline Hyperbilirubinemia & $690(86.0)$ & $118(52.9)^{* * *}$ & $808(78.8)$ & 338 (36.9)\#\#\# & $1146(59.0)$ \\
\hline Air leaking & $3(0.4)$ & $4(1.8)^{*}$ & $7(0.7)$ & $3(0.3)$ & $10(0.5)$ \\
\hline Surfactant & $103(12.8)$ & $28(12.6)$ & $131(12.8)$ & 86 (9.4)\# & $217(11.2)$ \\
\hline NIV / MV & 309 (38.5) & $95(42.6)$ & $404(39.4)$ & $370(40.4)$ & $774(39.9)$ \\
\hline $\mathrm{BPD}$ & $32(4.0)$ & $8(3.6)$ & $40(3.9)$ & 13 (1.4)\#\# & $53(2.7)$ \\
\hline NEC & $21(2.6)$ & $7(3.1)$ & $28(2.7)$ & 10 (1.1)\#\# & $38(2.0)$ \\
\hline $\mathrm{ROP}$ & $6(0.7)$ & $0^{*}$ & $6(0.6)$ & O\# & $6(0.3)$ \\
\hline Encephalopathy & $33(4.1)$ & $10(4.5)$ & $43(4.2)$ & 15 (1.6)\#\# & $58(3.0)$ \\
\hline In-hospital deaths & $33(4.1)$ & $23(10.3)^{* * *}$ & $56(5.5)$ & 31 (3.4)\# & $87(4.5)$ \\
\hline
\end{tabular}


Table 3 Perinatal status, morbidity and mortality rates of all hospitalized preterm infants in Huai'an (Continued)

\begin{tabular}{|c|c|c|c|c|c|}
\hline & \multicolumn{3}{|l|}{$\mathrm{HWCH}$} & \multirow{2}{*}{$\begin{array}{l}\text { Non-HWCH } \\
\text { hospitals }\end{array}$} & \multirow{2}{*}{$\begin{array}{l}\text { Whole } \\
\text { region }\end{array}$} \\
\hline & Inborn & Out-born & Total & & \\
\hline \multicolumn{6}{|c|}{ By postnatal days } \\
\hline $0-6$ & $20(2.5)$ & $15(6.7)^{* *}$ & $35(3.4)$ & $22(2.4)$ & $57(2.9)$ \\
\hline $7-27$ & $10(1.2)$ & $5(2.2)$ & $15(1.5)$ & $8(0.9) \#$ & $23(1.2)$ \\
\hline$\geq 28$ & $3(0.4)$ & $3(1.3)$ & $6(0.6)$ & $1(0.1)$ & $7(0.4)$ \\
\hline \multicolumn{6}{|c|}{ By GA weeks } \\
\hline $25-27$ & $8(1.0)$ & $5(2.2)$ & $13(1.3)$ & $9(1.0)$ & $22(1.1)$ \\
\hline $28-31$ & $15(1.9)$ & $10(4.5)^{*}$ & $25(2.4)$ & $13(1.4)$ & $38(2.0)$ \\
\hline $32-33$ & $6(0.7)$ & $3(1.3)$ & $9(0.9)$ & $3(0.3)$ & $12(0.6)$ \\
\hline $34-36$ & $4(0.5)$ & $5(2.2)^{*}$ & $9(0.9)$ & $6(0.7)$ & $15(0.8)$ \\
\hline
\end{tabular}

Abbreviations: CNY, Chinese Yuan (RMB); IVH, intraventricular hemorrhage; RDS, respiratory distress syndrome; PDA, patent ductus arteriosus; NIV / MV, noninvasive ventilation / mechanical ventilation; BPD, bronchopulmonary dysplasia; NEC, necrotizing enterocolitis; ROP, retinopathy of prematurity. Values are given as $\mathrm{n}, \mathrm{n}(\%)$, means $+\mathrm{SD}$ or median [interquartile ranges]. Percentage (\%) refers to number of total hospitalization in respective columns. ${ }^{*} P<0.05$, ** $P<0.01$ and *** $P<0.001$ for out-born vs. inborn of HWCH. \# $P<0.05$, \#\# $P<0.01$ and \#\#\# $P<0.001$ for non-HWCH vs. total of HWCH. For other definitions and abbreviations see Table 1 legends as reference

1. For definition of critical care see additional file 1

45]. As a result, we speculate that our understanding on the preterm birth status in China, either nation-wide or province-wide, would have substantial negligence, error and bias in terms of source and representativeness of data and database. We assumed that the Huai'an region represented the national average level of socioeconomic and healthcare status, and it may reflect the actual perinatal-neonatal healthcare style and quality in onefourth of sub-provincial prefectural regions in China. Our data showed approximately $4.5 \%$ of preterm birth rate and $10 \%$ preterm mortality rate. This may be translated into annually 650,000 preterm births and 65,000 deaths in China. The concept, methodology and data file of the current study may be generalizable to other regions, and served as a benchmark for future large-scale studies to explore perinatal-neonatal healthcare quality improvement, especially in the care of VPT and EPT population.

The prevalence of EPT (25-27 weeks of GA, 1.4\%o of total births, Table 2) was first reported from birth population-based survey data from China [11, 20], and was markedly lower than the $3-5 \%$ of total births in developed countries [3-9]. Despite the benefits provided by the universal health insurance policy for hospitalized delivery, especially for families with lower socioeconomic status, it remains controversial whether active prenatal and peripartum management including aggressive resuscitation at delivery, and neonatal critical care, should be provided to EPT in emerging regions in China. As deaths in livebirths below 28-week GA may be registered as abortion or stillbirth [19], or as neonatal death only after 7th of PND (unpublished domestic recommendation for birth registry since 2018), it can cause potential bias on vital statistics of the incidence/prevalence and outcome of preterm births and mortalities [7, 17, 18], leading to inaccurate estimation of the quality improvement of perinatal-neonatal care.

The comparison of preterm outcome between $\mathrm{HWCH}$ and non-HWCH admissions shed light on the role of main transferal centers in regional perinatal-neonatal network. As revealed in Tables 1 and 3, $\mathrm{HWCH}$ treated a disproportionally large part of high-risk pregnancies in the Huai'an region [20], and admitted more extremely and very preterm infants requiring critical care than other regional institutions. It implies a generous and effective in-utero transport policy taking place. It also supports a central role of $\mathrm{HWCH}$ in the regional perinatalneonatal healthcare, and indicates that investigation of the leading centers may help to understand the perinatal-neonatal healthcare at the regional level. Of note, the vast sub-provincial prefectural regions in China are estimated to account for more than $80 \%$ of annual national births (of 15-17 millions in 2014-2019). The inter-institutional difference in infrastructure should be narrowed with recent socioeconomic development, but quality of maternal-neonatal healthcare remains to vary widely. By including all birth data from level I-III institutions in the whole region of Huai'an [11,20], our study has overcome the limitations encountered in previous reports of vital statistics which often failed to account for prevalence and outcome of very and extreme preterm births [6, 17-19]. Based on the earlier nationwide collaborative studies, the quality of perinatal-neonatal healthcare at $\mathrm{HWCH}$ is likely to be above the national average level $[11,20,42,43]$ and the data file of outcome may be referred to for future inter-regional comparison to validate.

There was a trend that those with high or moderate risk of death by univariable regression analysis tended to have mild or no risk by multivariable Poisson regression model 
Table 4 Uni- and multivariable Poisson regression analysis of death risks of the whole Huai'an region

\begin{tabular}{|c|c|c|c|c|}
\hline \multicolumn{5}{|c|}{ A. The total preterm births } \\
\hline Variables & Category & $\begin{array}{l}\text { All-death } \\
\mathrm{n}(\%)\end{array}$ & $\begin{array}{l}\text { Univariable } \\
\text { RR (95\% Cl) }\end{array}$ & $\begin{array}{l}\text { Multivariable } \\
\text { RR ( } 95 \% \text { Cl) }\end{array}$ \\
\hline \multirow[t]{2}{*}{ Born in $\mathrm{HWCH}$} & No & $138(9.1)$ & & \\
\hline & Yes & $114(10.0)$ & $1.10(0.87-1.39)$ & $0.65(0.52-0.82)^{3}$ \\
\hline \multirow[t]{2}{*}{ PROM } & No & $192(10.5)$ & & \\
\hline & Yes & $60(7.3)$ & $0.69(0.53-0.92)^{1}$ & $1.09(0.88-1.35)$ \\
\hline \multirow[t]{2}{*}{$\mathrm{HDP}$} & No & $215(9.7)$ & & \\
\hline & Yes & $37(8.5)$ & $0.88(0.63-1.22)$ & $1.13(0.86-1.48)$ \\
\hline \multirow[t]{2}{*}{ GDM } & No & $239(9.5)$ & & \\
\hline & Yes & $13(10.6)$ & $1.12(0.66-1.90)$ & $1.39(0.92-2.12)$ \\
\hline \multirow[t]{2}{*}{ Anemia } & No & $228(10.0)$ & & \\
\hline & Yes & $24(6.4)$ & $0.64(0.43-0.96)^{1}$ & $0.84(0.63-1.12)$ \\
\hline \multirow[t]{2}{*}{ ANG } & No & $182(11.8)$ & & \\
\hline & Yes & $70(6.3)$ & $0.54(0.41-0.70)^{3}$ & $0.49(0.27-0.74)^{1}$ \\
\hline \multirow[t]{2}{*}{ Cesarean delivery } & No & $186(16.7)$ & & \\
\hline & Yes & $66(4.3)$ & $0.26(0.20-0.34)^{3}$ & $0.73(0.56-0.96)^{1}$ \\
\hline \multirow[t]{2}{*}{ Male } & No & $110(9.7)$ & & \\
\hline & Yes & $140(9.2)$ & $0.95(0.75-1.21)$ & $0.96(0.81-1.15)$ \\
\hline \multirow[t]{4}{*}{ GA, weeks } & $34-36$ & $63(3.5)$ & & \\
\hline & $32-33$ & $35(8.2)$ & $2.37(1.59-3.53)^{3}$ & $1.15(0.74-1.77)$ \\
\hline & $28-31$ & $93(29.6)$ & $8.58(6.38-11.5)^{3}$ & $1.66(1.10-2.51)^{1}$ \\
\hline & $25-27$ & $61(71.8)$ & $20.8(15.8-27.4)^{3}$ & $1.67(1.02-2.72)^{1}$ \\
\hline \multirow[t]{4}{*}{ BW, g } & $\geq 2500$ & $43(3.3)$ & & \\
\hline & $1500-2499$ & $81(7.5)$ & $2.25(1.57-3.23)^{3}$ & $0.96(0.65-1.41)$ \\
\hline & $1000-1499$ & $63(31.0)$ & $9.35(6.54-13.4)^{3}$ & $1.23(0.77-1.95)$ \\
\hline & $<1000$ & $65(94.2)$ & $28.4(21.0-38.3)^{3}$ & $2.25(1.38-3.67)^{2}$ \\
\hline \multirow[t]{2}{*}{ Multiple births } & No & $205(10.0)$ & & \\
\hline & Yes & $47(7.8)$ & $0.78(0.57-1.05)$ & $0.98(0.78-1.25)$ \\
\hline \multirow[t]{2}{*}{ SGA } & No & $199(7.9)$ & & \\
\hline & Yes & $24(20.5)$ & $2.58(1.76-3.78)^{3}$ & $1.09(0.79-1.51)$ \\
\hline \multirow[t]{2}{*}{ AF contamination } & No & $191(7.8)$ & & \\
\hline & Yes & $61(30.5)$ & $3.91(3.05-5.02)^{3}$ & $1.46(1.16-1.84)^{2}$ \\
\hline \multirow[t]{2}{*}{5 -min Apgar $\leq 7$} & No & $49(2.1)$ & & \\
\hline & Yes & $203(61.3)$ & $29.0(21.7-38.8)^{3}$ & $14.7(9.63-22.5)^{3}$ \\
\hline \multirow[t]{2}{*}{ Birth defects } & No & $219(8.5)$ & & \\
\hline & Yes & $33(40.7)$ & $4.78(3.57-6.40)^{3}$ & $1.73(1.28-2.33)^{3}$ \\
\hline \multicolumn{5}{|c|}{ B. The hospitalized preterm infants } \\
\hline Variables & Category & $\begin{array}{l}\text { In-hospital } \\
\text { deaths, n (\%) }\end{array}$ & $\begin{array}{l}\text { Univariable } \\
\text { RR (95\% Cl) }\end{array}$ & $\begin{array}{l}\text { Multivariable } \\
\text { RR (95\% CI) }\end{array}$ \\
\hline \multirow[t]{3}{*}{ Admitted in $\mathrm{HWCH}$} & No & $31(3.4)$ & & \\
\hline & Inborn & $33(4.1)$ & $1.22(0.75-1.97)$ & $0.96(0.58-1.58)$ \\
\hline & Out-born & $23(10.3)$ & $3.05(1.81-5.12)^{3}$ & $2.27(1.40-3.69)^{2}$ \\
\hline \multirow[t]{2}{*}{ Male } & No & $48(5.7)$ & & \\
\hline & Yes & $39(3.6)$ & $0.63(0.42-0.95)^{1}$ & $0.73(0.50-1.07)$ \\
\hline
\end{tabular}


Table 4 Uni- and multivariable Poisson regression analysis of death risks of the whole Huai'an region (Continued)

\begin{tabular}{|c|c|c|c|c|}
\hline \multirow[t]{4}{*}{ GA, weeks } & $34-36$ & $15(1.2)$ & & \\
\hline & $32-33$ & $12(3.3)$ & $2.90(1.37-6.14)^{2}$ & $1.49(0.68-3.26)$ \\
\hline & $28-31$ & $38(15.8)$ & $13.7(7.65-24.5)^{3}$ & $2.31(1.02-5.26)^{1}$ \\
\hline & $25-27$ & $22(55.0)$ & $47.7(26.8-84.9)^{3}$ & $2.44(0.90-6.61)$ \\
\hline \multirow[t]{4}{*}{ BW, g } & $\geq 2500$ & $9(1.1)$ & & \\
\hline & $1500-2499$ & $23(2.5)$ & $2.23(1.04-4.78)^{1}$ & $0.95(0.40-2.25)$ \\
\hline & 1000-1499 & $41(23.8)$ & $21.6(10.7-43.5)^{3}$ & $2.25(0.83-6.13)$ \\
\hline & $<1000$ & $14(70.0)$ & $63.3(31.1-128)^{3}$ & $3.66(1.16-11.6)^{1}$ \\
\hline \multirow[t]{2}{*}{ Cesarean delivery } & No & $58(7.5)$ & & \\
\hline & Yes & $29(2.5)$ & $0.33(0.21-0.51)^{3}$ & $0.53(0.34-0.83)^{2}$ \\
\hline \multirow[t]{2}{*}{5 -min Apgar $\leq 7$} & No & $47(2.6)$ & & \\
\hline & Yes & $40(24.0)$ & $9.04(6.12-13.4)^{3}$ & $2.73(1.75-4.26)^{3}$ \\
\hline \multirow[t]{2}{*}{ Birth defects } & No & $67(3.7)$ & & \\
\hline & Yes & $20(13.0)$ & $3.46(2.16-5.55)^{3}$ & $2.57(1.59-4.16)^{3}$ \\
\hline \multirow[t]{2}{*}{ Admitted within $24 \mathrm{~h}$ (after birth) } & No & $8(2.1)$ & & \\
\hline & Yes & $79(5.0)$ & $2.35(1.15-4.82)^{1}$ & $0.81(0.39-1.69)$ \\
\hline \multirow[t]{2}{*}{ RDS } & No & $29(1.7)$ & & \\
\hline & Yes & $58(21.2)$ & $12.2(7.97-18.7)^{3}$ & $2.76(1.65-4.65)^{3}$ \\
\hline \multirow[t]{2}{*}{ Pneumonia/sepsis } & No & $20(1.7)$ & & \\
\hline & Yes & $67(8.6)$ & $5.02(3.07-8.20)^{3}$ & $0.74(0.43-1.28)$ \\
\hline \multirow[t]{2}{*}{ NEC } & No & $72(3.8)$ & & \\
\hline & Yes & $15(39.5)$ & $10.4(6.62-16.4)^{3}$ & $1.76(1.03-3.03)^{1}$ \\
\hline \multirow[t]{2}{*}{ Surfactant } & No & $50(2.9)$ & & \\
\hline & Yes & $37(17.1)$ & $5.88(3.94-8.78)^{3}$ & $0.43(0.28-0.66)^{3}$ \\
\hline \multirow[t]{2}{*}{ NIV/MV } & No & $6(0.5)$ & & \\
\hline & Yes & $81(10.5)$ & $20.4(8.93-46.4)^{3}$ & $6.69(2.07-21.7)^{2}$ \\
\hline
\end{tabular}

Values are presented as number of deaths (\%) and corresponding relative risk (RR) and its $95 \%$ confidence intervals (95\% CI) by Poisson regression analysis. Multivariable analysis was done by all listed factors in the first column. For definition of all-death see Table 1 legends, note 6 ; for definition of in-hospital deaths see Table 1 legends, note 5; for other definitions and abbreviations see Tables 1 and 3 legends as reference

$1-3$, and stands for $P<0.05, P<0.01$ and $P<0.001$, respectively

(Table 4), well denoting that with decent obstetric management for high risk pregnancy and delivery, as well as NICU service quality, death risks of preterm birth and hospitalization may be effectively mitigated. As estimated by relative death risks of preterm birth and hospitalization through multivariable Poisson regression model, these should have explained, at least in part, the discrepancy of GA-specific mortality rates between birth and hospitalized population [7-11]. We therefore speculate that it should enable a comprehensive assessment of quality improvement in the regional perinatal-neonatal healthcare infrastructure as well as survival quality in follow-up of very and extremely preterm infants $[1-5,8,9]$.

The main limitation of the study was a relatively small sample size of EPT and VPT. However, as the first report of outcome of all hospitalized preterm infants derived from the total regional birth population, this study, on the other hand, has accounted for a large number of perinatal and postnatal risk factors as composite effects on the outcome measures from preterm birth to the whole hospitalization. Another limitation of the study was that no details of peripartum intervention were included for the perinatal risk analysis. Nevertheless, information on the prevalence of preterm births, morbidity and mortality by GA and efficacy of perinatal interventions may represent to a large part as a baseline of the regional perinatal-neonatal outcome, given the proportion of preterm infants requiring critical care and out-born infants transferal at $\mathrm{HWCH}$ and other level III hospitals. 


\section{Conclusion}

In conclusion, the prevalence of preterm birth and hospitalization based morbidity and mortality by GA strata or hospital categories, as characterized for both $\mathrm{HWCH}$ and non-HWCH, reflected the baseline and quality of healthcare status of regional network, representing the perinatal-neonatal healthcare in emerging regions. The concept and protocol of the study should be feasible to other regions of China and beyond to gain comprehensive understanding in the world-wide campaign for prevention of preterm birth.

\begin{abstract}
Abbreviations
aPMA: adjusted post menstrual age; BD: birth defects;

BPD: bronchopulmonary dysplasia; BW: birth weight; DR: delivery room: EPT: extreme preterm; GA: gestational age; HWCH: Huai'an Women and Children's Hospital; LPT: late preterm; MPT: moderate preterm;

MV: mechanical ventilation; NICU: neonatal intensive care unit; NIV: noninvasive ventilation; PND: postnatal day(s); RDS: respiratory distress syndrome; VPT: very preterm
\end{abstract}

\section{Supplementary Information}

The online version contains supplementary material available at https://doi. org/10.1186/s12884-021-03726-4

Additional file 1. Definitions of pregnancy and perinatal co-morbidities and complications and standard of care at admission and during hospitalization of preterm infants.

Additional file 2. A full list of members and their affiliations of Huai'an Perinatal-Neonatal study group.

Additional file 3: Table S1. Crude and gestational age-adjusted odds ratio $(O R)$ of death risks of the whole Huai'an region.

\section{Acknowledgements}

The authors thank all the committed staffs from member hospitals, as well as those at township hospitals and health care services, of the Huai'an Perinatal-Neonatal Study Group for generous participation and support of the study. Contribution of Drs. Y. Xu, MD and S. Luo, MD from Children's Hospital of Fudan University in data collection is highly appreciated. Dr. Guoyou Qin from the Department of Biostatistics, School of Public Health of Fudan University has reviewed all the data analysis and presentation, to which the authors are very grateful.

\section{Authors' contributions}

XG executed the study protocol, data collection and analysis, drafted and revised manuscript. XL and ZP supervised and validated clinical data collection and analysis, and reviewed manuscript. TQ, XZ and HW carried out data collection and analysis, and reviewed manuscript. YD engaged in data analysis, and critically revised manuscript. HY conceptualized and designed the study, coordinated and supervised data collection and analysis, and critically revised manuscript. BS conceptualized and designed the study, supervised data analysis, and critically revised manuscript. All authors approved the final manuscript as submitted and agree to be accountable for respective aspects of the work

\section{Funding}

Supported by grants from the Project of Maternal and Child Health Care by Jiangsu Provincial Commission of Health (F201402 [Yue H]), the National Natural Science Foundation (No. 81501288 [Dong Y]) and Shanghai Municipal Commission of Health (Project Young Physician Investigator [Dong Y]). The funding body had no influence on the design of the study, collection, analysis, and interpretation of data, and in writing the manuscript.

\section{Availability of data and materials}

The datasets used and/or analyzed during the current study are available from the corresponding author on reasonable request.

\section{Declarations}

Ethics approval and consent to participate

The ethics committee of Children's Hospital of Fudan University approved the study design and protocol, and waived the need for consent (\#2019194). This approval was adopted and approved by Huai'an Women and Children's Hospital and all participated hospitals in accordance with the Chinese regulations for clinical practice and investigation (www.nhc.gov.cn). The permissions and the names of the ethics committees and scientific committees (institutional review board) of the major participating hospitals are referred to Zhu X, et al. BMC Pregnancy Childbirth. 2019; 19(1): 224.

\section{Consent for publication}

Not applicable.

\section{Competing interests}

All the authors have declared no any financial and non-financial competing interests to disclose.

\section{Author details}

${ }^{1}$ The NCH Key Laboratory of Neonatal Diseases, National Children's Medical Center, Children's Hospital of Fudan University, Shanghai 201102, China. 2Department of Obstetrics, Huai'an Women and Children's Hospital, Huai'an 223002, Jiangsu, China. ${ }^{3}$ Department of Neonatology and Unit of Population Health Information, Huai'an Women and Children's Hospital, 104 South Ren Min Road, Huai'an 223002, Jiangsu, China. ${ }^{4}$ Unit of Population Health Information, Huai'an Women and Children's Hospital, Huai'an 223002, Jiangsu, China.

Received: 16 August 2020 Accepted: 17 March 2021

Published online: 10 April 2021

\section{References}

1. Costeloe KL, Hennessy EM, Haider S, Stacey F, Marlow N, Draper ES. Short term outcomes after extreme preterm birth in England: comparison of two birth cohorts in 1995 and 2006 (the EPICure studies). BMJ. 2012;345(dec04 3):e7976. https://doi.org/10.1136/bmj.e7976.

2. Ancel PY, Goffinet F, EPIPAGE-2 Writing Group, Kuhn P, Langer B, Matis J, et al. Survival and morbidity of preterm children born at 22 through 34 weeks' gestation in France in 2011: Results of the EPIPAGE-2 cohort study. JAMA Pediatr. 2015:169(3):230-8.

3. Norman M, Hallberg B, Abrahamsson T, Björklund LJ, Domellöf M, Farooqi A, et al. Association between year of birth and 1-year survival among extremely preterm infants in Sweden during 2004-2007 and 2014-2016. JAMA. 2019;321(12):1188-99. https://doi.org/10.1001/jama.2019.2021.

4. Bolisetty S, Legge N, Bajuk B, Lui K, New South Wales and the Australian Capital Territory neonatal intensive care Units' data collection. Preterm infant outcomes in New South Wales and the Australian Capital Territory. J Paediatr Child Health. 2015;51(7):713-21. https://doi.org/10.1111/jpc.12848.

5. Helenius K, Sjörs G, Shah PS, Modi N, Reichman B, Morisaki N, et al. Survival in very preterm infants: an international comparison of 10 national neonatal networks. Pediatrics 2017; 140 (6). pii: e20171264

6. He C, Liu L, Chu Y, Perin J, Dai L, Li X, et al. National and subnational all-cause and cause-specific child mortality in China, 1996-2015: a systematic analysis with implications for the sustainable development goals. Lancet Glob Health. 2017;5(2):e186-97. https:/doi.org/10.1016/S2214-109X(16)30334-5.

7. Manuck TA, Rice MM, Bailit JL, Grobman WA, Reddy UM, Wapner RJ, et al. Preterm neonatal morbidity and mortality by gestational age: A contemporary cohort. Am J Obstet Gynecol. 2016;215(1):103.e1-e14.

8. Stoll BJ, Hansen NI, Bell EF, Shankaran S, Laptook AR, Walsh MC, et al. Neonatal outcomes of extremely preterm infants from the NICHD neonatal research network. Pediatrics. 2010;126(3):443-56. https://doi.org/10.1542/ peds.2009-2959.

9. Haumont D, Modi N, Saugstad OD, Antetere R, NguyenBa C, Turner M, et al. Evaluating preterm care across Europe using the eNewborn European network database. Pediatr Res. 2020;88(3):484-95. https://doi.org/10.1038/s41390-020-0769-x. 
10. Sun L, Yue H, Sun B, Han L, Qi M, Tian Z, et al. Estimation of birth population-based perinatal-neonatal mortality and preterm rate in China from a regional survey in 2010. J Matern-Fetal Neonat Med. 2013;26(16): 1641-8. https://doi.org/10.3109/14767058.2013.794208.

11. Wang H, Yue H, Sun B, Zhu X, Niu H, Qi T, et al. Birth population survey in Huai'an in 2015: perinatal-neonatal mortality and preterm birth rate in emerging regions in China. J Matern-Fetal Neonatal Med. 2020;33(5):838-46. https://doi.org/10.1080/14767058.2018.1506439.

12. Sun B, Shao X, Cao Y, Xia S, Yue H. Neonatal-perinatal medicine in a transitional period in China. Arch Dis Child Fetal Neonatal Ed. 2013;98(5): F440-4. https://doi.org/10.1136/archdischild-2012-302524.

13. Li HT, Xue M, Hellerstein S, Cai Y, Gao Y, Zhang Y, et al. Association of China's universal two child policy with changes in births and birth related health factors: national, descriptive comparative study. BMJ. 2019;366:14680

14. Roberts D, Brown J, Medley N, Dalziel SR. Antenatal corticosteroids for accelerating fetal lung maturation for women at risk of preterm birth. Cochrane Database Syst Rev. 2017;3:CD004454

15. Travers CP, Carlo WA, McDonald SA, Das A, Bell EF, Ambalavanan N, et al. Mortality and pulmonary outcomes of extremely preterm infants exposed to antenatal corticosteroids. Am J Obstet Gynacol. 2018;218(1):130 e1-e13.

16. Althabe F, Belizán JM, McClure EM, Hemingway-Foday J, Berrueta M, Mazzoni A, et al. A population-based, multifaceted strategy to implement antenatal corticosteroid treatment versus standard care for the reduction of neonatal mortality due to preterm birth in low-income and middle-income countries: the ACT cluster-randomised trial. Lancet. 2015;385(9968):629-39. https://doi.org/10.1016/S0140-6736(14)61651-2.

17. Feng XL, Guo SF, Hipgrave D, Zhu J, Zhang L, Song L, et al. China's facilitybased birth strategy and neonatal mortality: a population-based epidemiological study. Lancet. 2011;378(9801):1493-500. https://doi.org/10.1 016/S0140-6736(11)61096-9.

18. Liang J, Mao M, Dai L, Li X, Miao L, Li Q, et al. Neonatal mortality due to preterm birth at 28-36 weeks' gestation in China, 2003-2008. Paediatr Perinat Epidemiol. 2011;25(6):593-600. https://doi.org/10.1111/j.1365-3016.2011.01232.x.

19. Zhu J, Liang J, Mu Y, Li X, Guo S, Scherpbier R, et al. Sociodemographic and obstetric characteristics of stillbirths in China: A census of nearly 4 million health facility births between 2012 and 2014. Lancet Global Health. 2016:4(2):e109-18.

20. Zhu X, Niu H, Wang H, Li X, Qi T, Ding W, et al. High risk pregnancy associated perinatal morbidity and mortality: a second birth populationbased survey in Huai'an in 2015. BMC Pregnancy Childbirth. 2019;19(1):224 https://doi.org/10.1186/s12884-019-2323-6.

21. World Health Organization. Born too soon: the global action report on preterm birth. Geneva. 2012:5.

22. Engle WA. A recommendation for the definition of "late preterm" (nearterm) and the birth weight-gestational age classification system. Semin Perinatol. 2006;30(1):2-7. https://doi.org/10.1053/j.semperi.2006.01.007.

23. Committee on Practice. ACOG Practice Bulletin No. 188: Prelabor rupture of membranes. Obstet Gynecol. 2018;131(1):e1-e14. https://doi.org/10.1097/A OG.0000000000002455

24. Committee on Practice. ACOG Practice Bulletin No. 202: gestational hypertension and preeclampsia. Obstet Gynecol. 2019;133(1):e1-e25

25. Committee on Practice. ACOG Practice Bulletin No. 190: gestational diabetes mellitus. Obstet Gynecol. 2018;131(2):e49-64. https://doi.org/10.1097/AOG. 0000000000002501

26. Sun D, McLeod A, Gandhi S, Malinowski AK, Shehata N. Anemia in pregnancy: a pragmatic approach. Obstet Gynecol Surv. 2017;72(12):730-7. https://doi.org/10.1097/OGX.0000000000000510.

27. Dorner RA, Burton VJ, Allen MC, Robinson S, Soares BP. Preterm neuroimaging and neurodevelopmental outcome: a focus on intraventricular hemorrhage, post-hemorrhagic hydrocephalus, and associated brain injury. J Perinatol. 2018; 38(11):1431-43. https://doi.org/10.1038/s41372-018-0209-5.

28. Sweet DG, Carnielli V, Greisen G, Hallman M, Ozek E, Plavka R, et al. European consensus guidelines on the management of neonatal respiratory distress syndrome in preterm infants-2013 update. Neonatology. 2013; 103(4):353-68. https://doi.org/10.1159/000349928.

29. Committee on Fetus and Newborn, Adamkin DH. Postnatal glucose homeostasis in late-preterm and term infants. Pediatrics. 2011;127(3):575-9. https://doi.org/10.1542/peds.2010-3851.

30. American Academy of Pediatrics Subcommittee on Hyperbilirubinemia. Management of hyperbilirubinemia in the newborn infant 35 or more weeks of gestation. Pediatrics. 2004;114(1):297-316. https://doi.org/10.1542/peds.114.1.297.
31. Jobe $A H$, Bancalari E. Bronchopulmonary dysplasia. Am J Respir Crit Care Med. 2001;163(7):1723-9. https://doi.org/10.1164/ajrccm.163.7.2011060.

32. Bell MJ, Ternberg JL, Feigin RD, Keating JP, Marshall R, Barton L, et al. Neonatal necrotizing enterocolitis. Therapeutic decisions based upon clinical staging. Ann Surg. 1978;187(1):1-7. https://doi.org/10.1097/ 00000658-197801000-00001

33. Fierson WM, American Academy of Pediatrics Section on Ophthalmology, American Academy of Ophthalmology, American Association for Pediatric Ophthalmology and Strabismus, American Association of Certified Orthoptists. Screening examination of premature infants for retinopathy of prematurity. Pediatrics. 2018;142(6):e20183061.

34. Parikh $P$, Juul SE. Neuroprotection strategies in preterm encephalopathy. Semin Pediatr Neurol. 2019;32:100772. https://doi.org/10.1016/j.spen.2019.08.008.

35. World Health Organization. International statistical classification of disease and related health problems. 10th revision (ICD-10), Geneva 1993.

36. Ballard JL, Khoury JC, Wedig K, Wang L, Eilers-Walsman BL, Lipp R. New Ballard Score, expanded to include extremely premature infants. J Pediatr. 1991;119(3):417-23.

37. Fenton TR, Kim JH. A systematic review and meta-analysis to revise the Fenton growth chart for preterm infants. BMC Pediatr. 2013;13(1):59. https:// doi.org/10.1186/1471-2431-13-59.

38. Smithhart W, Wyckoff MH, Kapadia V, Jaleel M, Kakkilaya V, Brown LS, et al. Delivery room continuous positive airway pressure and pneumothorax. Pediatrics 2019; 144 (3). pii: e20190756.

39. Dargaville PA, Gerber A, Johansson S, De Paoli AG, Kamlin CO, Orsini F, et al. Incidence and outcome of CPAP failure in preterm infants. Pediatrics. 2016; 138(1):e20153985. https://doi.org/10.1542/peds.2015-3985.

40. Gordis L. Mortality rates in: epidemiology $5^{\text {th }}$ edition. Philadelphia: Elsevier Saunders; 2013. p. 64-5.

41. Callaghan WM, MacDorman MF, Shapiro-Mendoza CK, Barfield WD. Explaining the recent decrease in US infant mortality rate, 2007-2013. Am J Obstet Gynacol. 2017;216(1):73.e1-8.

42. Wang H, Gao X, Liu C, Yan C, Lin X, Yang C, et al. Morbidity and mortality of neonatal respiratory failure in China. Surfactant treatment in very immature infants. Pediatrics. 2012;129(3):e731-40. https://doi.org/10.1542/peds.2011-0725.

43. Wang H, Gao X, Liu C, Yan C, Lin X, Dong Y, et al. Surfactant reduced the mortality of neonates with birth weight $>1500 \mathrm{~g}$ and hypoxemic respiratory failure: a survey from an emerging NICU network. J Perinatol. 2017;36:883-9.

44. Kong X, Xu F, Wu R, Wu H, Ju R, Zhao X, et al. Neonatal mortality and morbidity among infants between 24 to 31 complete weeks: a multicenter survey in China from 2013 to 2014. BMC Pediatr. 2016;16(1):174. https://doi. org/10.1186/s12887-016-0716-5.

45. Wu F, Liu G, Feng Z, Tan X, Yang C, Ye X, et al. Short-term outcomes of extremely preterm infants at discharge: a multicenter study from Guangdong province during 2008-2017. BMC Pediatr. 2019;19(1):405. https://doi.org/10.1186/s12887-019-1736-8.

\section{Publisher's Note}

Springer Nature remains neutral with regard to jurisdictional claims in published maps and institutional affiliations.

Ready to submit your research? Choose BMC and benefit from:

- fast, convenient online submission

- thorough peer review by experienced researchers in your field

- rapid publication on acceptance

- support for research data, including large and complex data types

- gold Open Access which fosters wider collaboration and increased citations

- maximum visibility for your research: over $100 \mathrm{M}$ website views per year

At $\mathrm{BMC}$, research is always in progress.

Learn more biomedcentral.com/submission 